International Journal of Medical Arts 2020; 2 [3]: 503-507.

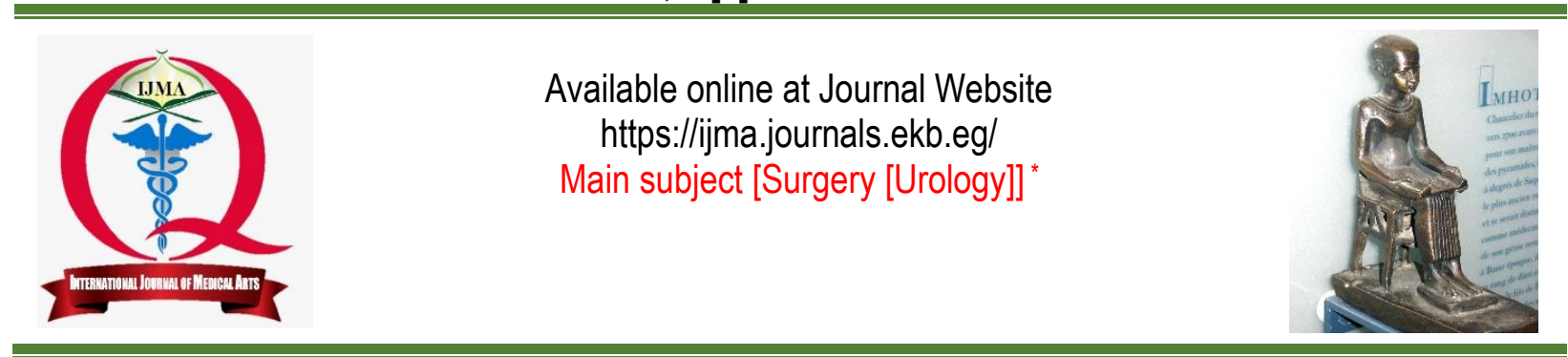

Case-Series

\title{
Urolithiasis in Post Renal Transplant Recipients: Case series
}

\author{
Nabeel Joda Kuwaijo [a]; Ponnusamy Mohan[b]; Mohammed Sayed Aboelmagd[c]; Sadiq Lala[d] \\ Department of Urology, The Galway Clinic, Royal College of Surgeons, Ireland [a] . \\ Department of Urology, Andrology \& Transplant, Beaumont Hospital, Beaumont Road, Dublin, Ireland ${ }^{[\mathbf{b}]}$ \\ Department of Urology, Mater Misericordiae University Hospital, Dublin, Ireland [c] \\ Department of Urology and Transplant, The Royal Hospital, Muscat, Oman [d] \\ Corresponding author: Nabeel Joda Kuwaijo \\ Email:dr_kwaijo@yahoo.com
}

Received at: March 31, 2020; Revised at: June 2, 2020; Accepted at: June 3, 2020; Available online at: June 3, 2020

DOI: 10.21608/ijma.2020.27026.1118

\section{ABSTRACT}

Renal transplantation represents the optimal management option for end-stage renal disease. it is associated with favourable outcome. Urolithiasis after renal transplant is an extremely rare condition, with an incidence less than $1 \%$ after renal transplantation.

We reported six post renal transplant patients who had renal stones. The stones located in the transplanted kidney, its ureter or both. Transplanted kidney and ureteric stones had been reported $2-3$ years after transplantation, while the urinary bladder calculi reported 8 - 10 years after transplantation, at the site of ureteral implantation to the bladder over the site of sutures and in the absence of any post-transplant complications. A written consent has been signed by the patient to release information for this study. The treatment was individualized for each patient. Interestingly, one patient with asymptomatic post-transplant renal stone needs no intervention "we watched and see" and he passed the stone within shortest follow up period that confirmed by ultrasound examination. Others, underwent extracorporeal wave lithotripsy or percutaneous nephrostomy and antegrade double-J stent insertion.

Keywords: Renal transplant; Vesical calculus; Suture; Stone; Ureter

This is an open access article under the Creative Commons license [CC BY] [https://creativecommons.org/licenses/by/2.0/]

Please cite this article as: Kuwaijo NJ, Mohan P, Aboelmagd MS, Lala S. Urolithiasis in Post Renal Transplant Recipients: Case series. IJMA 2020; 2[3]: 503-507.

* Main subject and any subcategories have been classified according to research topic. 


\section{INTRODUCTION}

Renal transplantation is now considered as the best and long-term treatment option for patients with end-stage renal disease. The number of performed renal transplant had been consistently and markedly increased worldwide since the $1990 \mathrm{~s}^{[1]}$. When non complicated kidney transplant recipient patient develop urolithiasis, it can be a challenge deciding whether these patients should receive endoscopic urological intervention or deemed suitable for treatment with lithotripsy plus or minus radiologic intervention[2].

Renal stones after transplantation had higher risk of marked morbidity due to obstruction, sepsis or relative loss of allograft function. These stones usually asymptomatic and usually discovered accidentally during imaging studies. This could explain the delayed diagnosis of such condition after transplantation. The early diagnosis of such condition is essential, as the patient is relying on a single kidney. The asymptomatic nature of posttransplantation stone is not a contra-indication to urologic intervention. Cicerell et al. [3] reported that, stone in patients with solitary transplanted kidney, must be removed in each patient as it may lead to urinary infection or migrated to ureter causing obstruction with acute renal failure. Oliveira et al. ${ }^{[4]}$ reported that, post-transplantation stones can be treated with observation, shock wave lithotripsy, endoscopic, percutaneous or open surgery. However, each of these approaches had its advantages and disadvantages or even limitations. Thus, the treatment option must be individualized for each patient[5].

At the time of presentation of our patients, we did not have guidelines on how to manage those categories. Here, we present six cases of post renal transplant patients with stones [developed at 2-3 years in the kidney and ureter and 9 years in the bladder over the stitch thread after renal transplantation]. Our patients did not have any complication at the time of or after transplantation surgery. We could not attribute the development of vesical calculus 9 years after transplantation to any other medical or surgical causes. The nest or the nidus of the calculus appeared to be developed on the sutures used at the site of ureterovesical anastomosis. In the next sections, we will provide a short description of each of reported cases.

\section{Case number one: Transplanted Ureteric} calculus: [Living Related Donor Transplantation]

A female patient, 29 years old, referred by nephrologist. Here compliant was that of dysuria and lower right abdominal pain of 3 months. She is never smoke nor drink alcohol; she is working as a clerk, living with her husband in their own accommodation. She underwent living related kidney transplantation by our team, for end stage renal disease secondary to glomerular diseases. There was no posttransplant complication, subsequently as all transplant patients, she underwent regular follow up with the nephrologist. She remained asymptomatic and maintained normal renal functions throughout.

She underwent right hemi thyroidectomy 3 years after transplant for excision of right thyroid nodule, the histopathology reported as benign hyperplasia and her thyroid function test is normal. She is hypertensive, controlled with medications. No known allergies, currently she is not diabetic, no past surgical history [other than transplantation and thyroidectomy], and not on chronic medication other than immunosuppressant medication and antihypertensive medications.

Until 4 years after transplantation, she developed unexplained and persistent lower urinary tract symptoms with negative urine culture, she had no benefit from symptomatic treatment with frequent attacks of right lower abdominal pains and graft tenderness. The pain started in the right iliac fossa, sudden onset pain, dull in nature, lasting for few hours' duration, deep seating, radiating to the suprapubic area and upper right thigh, exacerbated by drinking plenty of fluid especially after coffee and tea. There were no other related symptoms like nausea nor vomiting. Urine analysis revealed persistent white blood cells [WBC] and red blood cells $[R B C]$. Other laboratory investigations were within normal reference ranges. Her ultrasonography showed moderate dilatation of transplanted ureter and renal pelvis with resistance index [RI] of 0.7$]$. The plain X- Ray showed the presence of radioopaque shadow in the right iliac fossa, which was confirmed by non-contrast computed tomography [CT] scan to have a $9 \mathrm{~mm}$ in diameter stone in the distal transplanted ureter.

Treatment and progress: We discussed the possible treatment options with the patient after we had discussed her case in our oncology-stone- 
multidisciplinary meeting; she opted for lithotripsy which had been resulted in partial fragmentation. Thereafter; the patient developed sustained pain in the right iliac fossa, her serum creatinine raised to $220 \mathrm{umol} / \mathrm{L}$. We performed urgent ultrasonography which showed increase in the hydrouretronephrosis of the transplanted kidney. Immediately she was consented for percutaneous nephrostomy and antegrade double-J stent insertion [Figure 1]. The procedure had been carried out by an interventional radiologist uneventfully. The nephrostogram showed moderate hydrouretronephrosis with smooth filling defect at the distal right ureter suggestive of calculus. Subsequently, she underwent extracorporeal shock wave lithotripsy [ESWL] with $\mathrm{JJ}$ stent in-situ this resulted with complete clearance of the stone with Storz lithotripter. JJ stent was removed after 6 weeks of insertion. Patient was discharged to her nephrologist with normal renal function for continuation of the care and follow up.

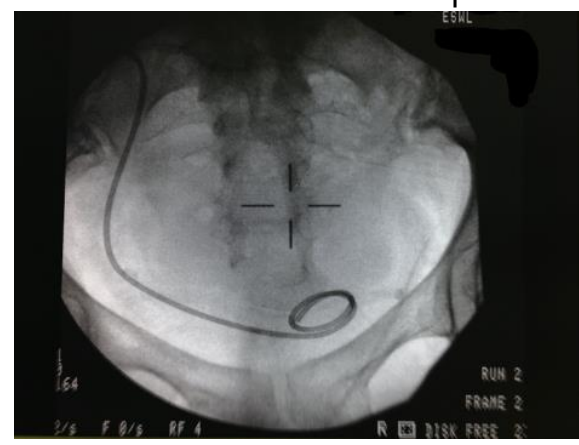

Figure [1]: Fluoroscopic image [ESWL]

Case number two: Transplanted kidney and ureteric calculi [living non-related kidney transplant]:

Here, a 22 years old male underwent living nonrelated kidney transplantation abroad, with regular follow up with nephrologist. He was presented by dysuria and haematuria three years after renal transplantation. He was hypertensive, had dyslipidaemia and hyperuricemia controlled with medications. He was not diabetic and had no positive past surgical history [other than transplantation]. His Serum creatinine was marginally raised to 122 umol/L, with elevated serum uric acid [up to 536 umol/L]. Other laboratory investigations were within normal ranges. Ultrasonography revealed 6-mm stone in the transplanted kidney with tortuous and dilated transplanted ureter up to lower third. There were another two ureteric stones each measuring 6 $\mathrm{mm}$ in diameter, with normal resistive index, and DTPA renogram was normal.
Treatment and progress: The lower ureteric stones were treated successfully with 2 sessions of ESWL with Siemens lithotripter. Thereafter, the patient passed the stones achieving stone free status and discharged with normal serum creatinine to be followed with nephrologist. No treatment intervention was required for renal stone. During the course of treatment with ESWL patient passed a fragment of stone, which had been sent for analysis and was composed of calcium oxalate. No uric acid crystals were found in spite of hyperuricemia at the time of diagnosis. The patient was on Allopurinol and the stones where radio opaque. Uric acid levels were within normal [with treatment] during the follow ups.

Case number 3: Transplanted Ureteric calculus [living non-related donor]:

We presented an 18 years old male, with history of controlled - insulin dependent diabetes mellitus type 1 and chronic kidney failure. He is hypertensive controlled with medications; he did not have neither dyslipidaemia nor hyperuricemia, no past surgical history before the current one. He underwent kidney transplant from a living non-related donor abroad, had peaceful four years after his transplantation until he started to have acute right iliac and abdominal pain with haematuria. In addition, he had mild dysuria over two weeks. His laboratory investigations were normal along with good control of blood glucose levels. Ultrasound scan showed $10 \mathrm{~mm}$ diameter stone in the lower end of the transplanted ureter with mild dilatation of transplanted ureter and normal resistive index.

Treatment and progress: The lower ureteric stone treated successfully with semirigid ureteroscopy and laser fragmentation with $\mathrm{JJ}$ stent insertion, stone free status, discharged from urology service after JJ stent removal after couple of weeks.

Cases number 4 and 5: Vesical calculus at the surgical transplantation site of a ureter in the bladder [cadaveric donor kidney transplant]:

Here were presented two patients, a 55 years old female and a 33 years old male, complained of bladder irritative symptoms [frequency, urgency, feeling of incomplete emptying of the bladder, dysuria and burning micturition] with negative urine culture. The patients underwent cadaveric non related donor kidney transplantation abroad. Our male patient, 8 years after transplantation, was 
symptom free. However, he had an irregular radioopaque shadow at the bladder area on X-ray KUB [Figure 2]. Ultrasound examination revealed a 9-mm, non-floating [with position change] bladder mass [Figure 3]. The presented data are mainly for our male patients. However, the data regarding the female patient did not differ, and she underwent similar treatment and followed nearly a similar course. Thus, reiteration of data was not provided.

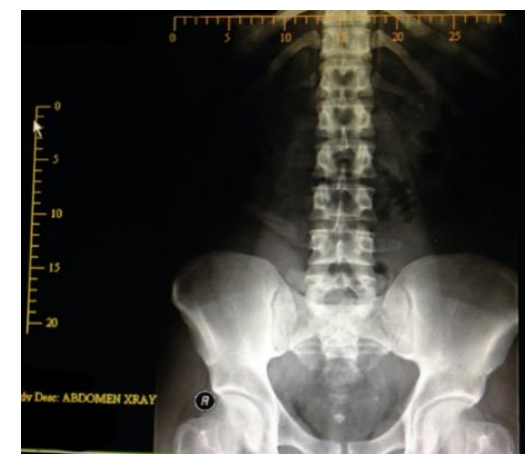

Figure [2]: KUB X-ray showing radio opaque stone in the bladder region

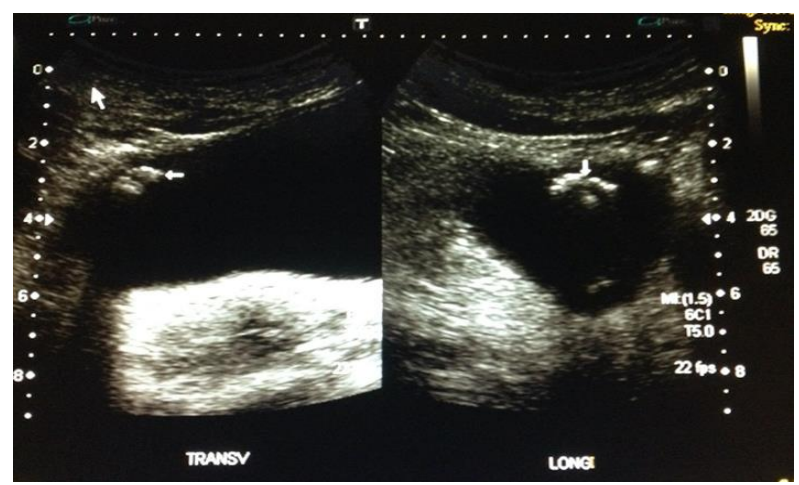

Figure [3]: Ultrasound showing the stone in the bladder which not float with changing position

The patient was posted for rigid cystoscopic examination. At cystoscopy, the urethra was normal, prostate wasn't obstructing with normal bladder neck. At the site of neo-ureter, there was a stitch with calcification this was grasped gently as seen in next figures [4 through 7].

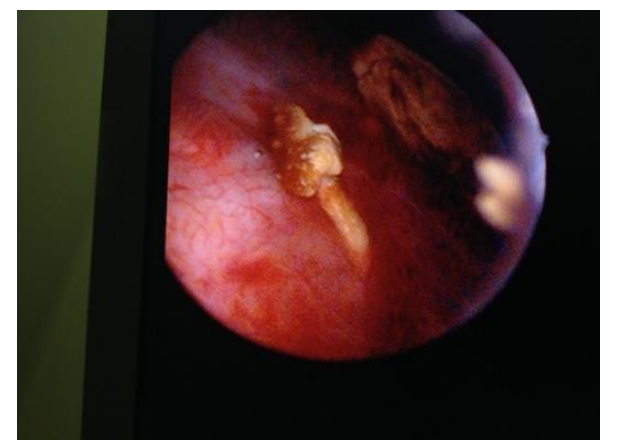

Figure [4]: The cystoscopy appearance of the thread with stone at the tip with larger stone

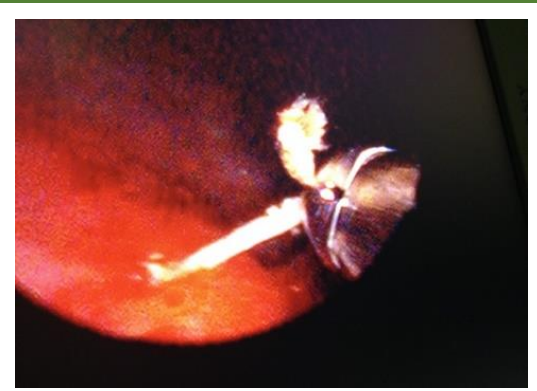

Figure [5]: The thread being pulled out the bladder wall with forceps pulling it

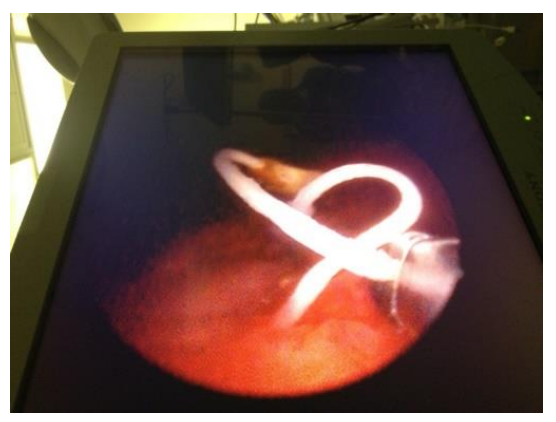

Figure [6]: The thread being pulled out the bladder wall with forceps

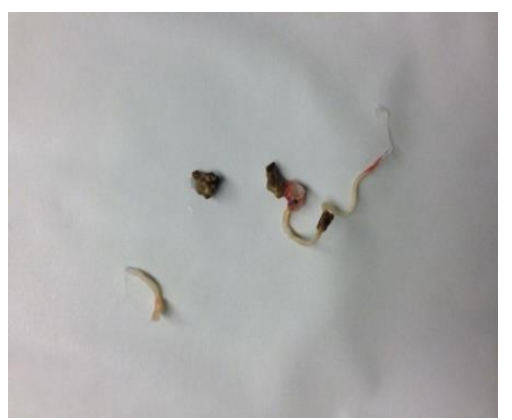

Figure [7]: the calculus with its nidus [the sutures]

Case number 6: Tiny renal calculus in the upper pole of the transplanted kidney [Living Related Donor Transplantation]:

A 50-years-old male, four-years post-live related kidney donor transplantation was referred by his nephrologist for an opinion on an asymptomatic, 2 $\mathrm{mm}$ stone in the upper pole of the transplanted kidney discovered accidentally on an ultrasound scan during routine assessment in his follow-up visit. He is type- 2 diabetic, well-controlled, and his laboratory tests showed stability compared to his baseline tests. It was thoroughly discussed with the patient and we both agreed to monitor this tiny stone; and the agreed observation period was safe initial management option. The patient passed the stone in 4 weeks and the stone, and six weeks follow up ultrasound scan was clear. Patient continue his follow up as usual. 


\section{DISCUSSION}

Although it is a rare complication, calculi formation following renal transplantation are reported everywhere and then.

The first report of vesical calculus after a renal transplant at the site of ureteroneocystostomy was reported at 1975 by O'Dea MJ et al.[6]. An interesting observation in some of our cases is the absence of any symptoms related to the stone development after transplantation. This was ascribed to denervation of transplanted graft [7-8].

In the current case series, patients were usually treated by ESWL or even observation [as in the last case]. Bhadauria et al. [9] reported two cases of posttransplant renal stones. Both were managed by ESWL. Other researchers described the use of PCNL after transplantation with preservation of renal unction and low morbidity[7].

Burgher et al.[10] advocated cautious observation as an alternative treatment option. Kang et al.[11] also recommended watchful waiting for asymptomatic renal stones.

The vesical stone formation is an extremely rare finding. In 1999, Rhee BK et al.[12] reported four cases of vesical stones following combined pancreas/renal transplantation, where calculus formed on an exposed suture. However, they did not find vesical calculi in patients with isolated renal transplantation. We could not be attribute to any medical or surgical cause for formation of vesical calculus which was attached to exposed stitch 8 to 10 years after transplantation, mostly that exposed part of stitch acted as a nidus for stone formation.

To summarize, our patients had no posttransplant complications with normal renal function and absent risk factors for stone formations. However, it not excludes stone formation. Thus, ultrasound follow up examination on an annual basis is recommended. This permits early diagnosis and treatment if indicated. In addition, careful renal function monitoring is essential.

\section{Financial and Non-Financial Relationships and Activities of Interest}

None declared by the author

\section{REFERENCES}

1. Emiliani E, Subiela JD, Regis R, Angerri O, Palou J. Over 30-yr Experience on the Management of Graft Stones After Renal Transplantation. Eur Urol Focus 2018 Mar;4[2]:169-174. [DOI: 10.1016/j.euf.2018.06.007].

2. Mahdavi R, Tavakkoli M, Taghavi R, Ghoreifi A. Minimally invasive procedures for treatment of urolithiasis in transplanted kidneys. Exp Clin Transplant. 2014; 12 [3]: 200-204. [DOI: 10.6002/ect.2013.0297].

3. Cicerello E, Merlo F, Maccatrozzo L. Management of Clinically Insignificant Residual Fragments following Shock Wave Lithotripsy. Adv Urol. 2012; 2012:320104. doi:10.1155/2012/320104

4. Oliveira M, Branco F, Martins L, Lima E. Percutaneous nephrolithotomy in renal transplants: a safe approach with a high stone-free rate. Int Urol Nephrol 2011; 43:329-335. [DOI: 10.1007/s11255-010-9837-1].

5. Tonyali S, Aydin AM. Evaluation of Deceased Kidney Donors for Renal Stone Disease: Is Computed Tomography Needed? Curr Urol 2017; 11:113-116. [DOI: 10.1159/000447204].

6. O'Dea MJ, Zincke H, Rivers TA, Leary FG. Occurrence of vesical calculus following renal transplantation. $\mathrm{Br} \mathrm{J}$ Urol 1975; 47:424. [DOI: 10.1111/].1464-410x.1975.tb 04000.x].

7. Lu HF, Shekarriz B, Stoller ML. Donorgifted allograft urolithiasis: early percutaneous management. Urology 2002; 59:25. [DOI: 10.1016/s0090-4295[01]01490-x].

8. Ferreira Cassini M, Cologna AJ, Ferreira Andrade M, Lima CJ, Medeiros Albuquerque U, Pereira Martins AC, Tucci Junior S. Lithiasis in 1,313 kidney transplants: incidence, diagnosis, and management. Transplant Proc. 2012; 44[8]:2373-2375. [DOI: 10.1016/j. transproceed. 2012.07.052].

9. Bhadauria RP, Ahlawat R, Kumar RV, Srinadh ES, Banerjee GK, Bhandari M. Donor-gifted allograft lithiasis: extracorporeal shockwave lithotripsy with over table module using the Lithostar Plus. Urologia Internationalis. 1995; 55 [1]: 51-55. [DOI:10.1159/ 000282750].

10. Burgher A, Beman M, Holtzman JL, Monga M. Progression of nephrolithiasis: long-term outcomes with observation of asymptomatic calculi. J Endourol. 2004; 18:534. [DOI: 10.1089/end.2004.18.534].

11. Kang HW, Lee SK, Kim WT, Kim YJ, Lee SC, Kim WJ. Natural history of asymptomatic renal stones and prediction of stone related events. J Urol. 2013; 189:1740-1746. [DOI: 10.1016/j.juro. 2012.11.113].

12. Rhee BK, Bretan PN, Stoller ML. Urolithiasis in renal and combined pancreas/renal transplant recipients. J Urol 1999; 1161:1458-1462. [PMID: 10210372]. 